\title{
From bed-side to web-side, Advanced-Telemedicine platform for Spinal Muscular Atrophy
}

David korn ${ }^{1}$, Beatrice Berti ${ }^{1}$, Andrea Cambieri ${ }^{1}$, giovanni scambia ${ }^{1}$, paolo sergi ${ }^{1}$, emilio meneschincheri ${ }^{1}$, daniela leone ${ }^{1}$, maria carmela pera ${ }^{1}$, concetta palermo ${ }^{1}$, Danilo Buonsenso $^{2}$, Roberta Onesimo ${ }^{3}$, Cristina De Rose ${ }^{4}$, Marika Pane ${ }^{3}$, and Eugenio Mercuri ${ }^{3}$

${ }^{1}$ Policlinico Universitario Agostino Gemelli Dipartimento Scienze della Salute della Donna e del Bambino

${ }^{2}$ Ospedale Pediatrico Bambino Gesu

${ }^{3}$ Policlinico Universitario Agostino Gemelli

${ }^{4}$ Universita Cattolica del Sacro Cuore Sede di Roma

September 25, 2021

\begin{abstract}
We aimed to assess feasibility, accuracy, satisfaction of an advanced-telemedicine (A-TM) platform designed for remote physical evaluation, especially focused on lung auscultation, in spinal muscular atrophy (SMA) patients. Children affected by type 1 and 2 SMA, typically present generalized weakness, scoliosis, chest deformities the leading cause of progressive respiratory insufficiency and recurrent hospitalization. Covid-19 stimulated efforts to adopt innovative digital health solutions especially when caring for people living with disabilities. Because of chest asymmetry and scoliosis, SMA patients are not always the ideal candidates for telemedicine tools that have proved to be useful in the general population. 23 children affected by SMA (15 type 1 and 8 type 2) with different degree of scoliosis and chest asymmetry. Prospective study: We localized optimal thoracic auscultatory landmarks with traditional stethoscope and lung ultrasound for each child. Carers were trained to record complete lung auscultation independently and share data with our physicians via A-TM platform. After the first remote exam, carers videorecorded their experience (satisfaction). Our physicians blindly rated the audio files shared via A-TM which were compared to traditional auscultation findings for each child. to assess. Overall feasibility and accuracy of carers-performed remote physical evaluation. Our study showed that remotely performed lung auscultation was possible in all type 1 and 2 SMA children but adaptations to find optimal landmarks were needed in cases with asymmetrical or rotated chest and trunk. A-TM tools may simplify access to care, reduce logistic/economic burden for families, improve communication, safety and disease management while limiting infection exposure.
\end{abstract}

\section{From bed-sideto web-side, Advanced-Telemedicine platform for Spinal Muscular Atrophy}

David Korn $\mathrm{MD}^{1, *}$, Beatrice Berti $\mathrm{MD}^{2, *}$, Daniela Leone $\mathrm{PhD}^{2}$, Concetta Palermo $\mathrm{PhD}^{2}$, Danilo Buonsenso $\mathrm{MD}^{1,3}$, Roberta Onesimo $\mathrm{PhD}^{1}$, Cristina De Rose $\mathrm{MD}^{1}$, Maria Carmela Pera $\mathrm{PhD}^{2,3}$, Andrea Cambieri $\mathrm{MD}^{4}$, Emilio Meneschincheri ${ }^{5}$, Paolo Sergi ${ }^{5}$, Giovanni Scambia $\mathrm{MD}^{2,6,3}$, Marika Pane $\mathrm{PhD}^{2,3}$ and Eugenio Mercuri $\mathrm{PhD}^{2,3}$.

1 Pediatric Unit, Fondazione Policlinico Universitario A. Gemelli IRCCS, Rome, Italy.

2 Pediatric Neurology Unit and Centro Clinico Nemo, Fondazione Policlinico Universitario A. Gemelli IRCCS, Rome, Italy.

3 Catholic University of Rome, Italy. 
4 Healthcare Management - Fondazione Policlinico Universitario Agostino Gemelli -IRCCS, Rome, Italy.

5 ICT management Fondazione Policlinico Universitario Agostino Gemelli -IRCCS, Rome, Italy.

6 Ginecology Oncology Unit, Fondazione Policlinico Universitario Agostino Gemelli -IRCCS, Rome, Italy.

* Both first authors, contributing equally to this paper.

\section{Corresponding Authors}

Eugenio Mercuri,

Pediatric Neurology Unit

Fondazione Policlinico Universitario A. Gemelli IRCCS, Largo Gemelli 00168, Roma, Italy

Tel.: +3906 30155340; fax: +390630154363.

E-mail address:

eugeniomaria.mercuri@unicatt.it

Keywords: Spinal Muscular Atrophy, Advance-Telemedicine platforms, Covid-19, Chest and spine deformities

Running title: Advanced-Telemedicine platform for Spinal Muscular Atrophy

Conflicts of Interest and Source of Funding: All authors declare that they have not conflict of interest.

Abstract

Objective: To assess feasibility, accuracy, satisfaction of an advanced-telemedicine (A-TM) platform designed for remote physical evaluation, especially focused on lung auscultation, in spinal muscular atrophy (SMA) patients. Children affected by type 1 and 2 SMA, typically present generalized weakness, scoliosis, chest deformities the leading cause of progressive respiratory insufficiency and recurrent hospitalization. Covid-19 stimulated an effort to adopt innovative digital health solutions especially when caring for people living with disabilities. Because of chest asymmetry and scoliosis, SMA patients are not always the ideal candidates for telemedicine tools that have proved to be useful in the general population.

Design: Prospective study.

Patients: 23 children affected by SMA (15 type 1 and 8 type 2) with different degree of scoliosis and chest asymmetry.

Methodology: We localized optimal thoracic auscultatory landmarks with traditional stethoscope and lung ultrasound for each child. Carers were trained to record complete lung auscultation independently and share data with our physicians via A-TM platform. After the first remote exam, carers videorecorded their experience (satisfaction). Our physicians blindly rated the audio files shared via A-TM which were compared to traditional auscultation findings for each child. to assess. Overall feasibility andaccuracy of carers-performed remote physical evaluation.

Results: Our study showed that remotely performed lung auscultation was possible in all type 1 and 2 SMA children but adaptations to find optimal landmarks were needed in cases with asymmetrical or rotated chest and trunk.

Conclusion : A-TM tools may simplify access to care, reduce logistic/economic burden for families, improve communication, safety and disease management while limiting infection exposure.

(249w)

Introduction 
Spinal Muscular Atrophy is a rare neuromuscular disorder caused by autosomic recessive mutations in the Survival Motor Neuron 1 (SMN1) gene. According to age of onset and clinical severity, SMA is associated with different pediatric forms, among them type 1 and 2 are the most severe ones [1]. The weakness classically also involves the respiratory muscles with a typical diaphragmatic breathing pattern, most obvious in type 1 infants with subsequent need for mechanical ventilation support, recurrent hospitalization and, before treatment became available, premature exitus $[2,3]$.

The advent of new therapies increasing SMN protein either by gene replacement or incrementing SMN2 mRNA splicing, has produced a dramatic change in progression of the disease, improvement in survival, motor and respiratory function, more obvious in type 1 infants, who, until the advent of therapy, rarely survived beyond the 2nd year and never achieved independent sitting $[4,5,6]$.

With increased survival it has become even more important to follow these patients over time. According to the SMA International Standard-of-Care (SoC), the assessments should be repeated every 3 to 6 months, involving a multidisciplinary team of specialists at tertiary centers for neuromuscular diseases, for routine in-personassessment and bed-side physical evaluations [2, 3].

Unfortunately, as a result of Covid-19 pandemic, the physical access to care, as well as our chance to guarantee the SoC, became more difficult since many tertiary centers had to limit routine in-person evaluation, focus resources and reduce the risk of infection. In order to address some of the difficulties faced by families to attend routine assessments, we first developed a "contactless" patient video-monitoring platform [7] to assure a safe, privacy compliant and reliable access to specialists while providing, through questionnaires and tutorials, some essential care, including rehabilitation, that could not be otherwise guaranteed during the pandemic. While the platform proved to be extremely useful to replace some of the routine follow up assessments, SMA families were still very concerned about the difficulties experienced in getting physical examinations during acute events. Several parents reported that in the last few months "red flags" of respiratory infections, happened to go unnoticed or underestimated by themselves or local practitioners, who had little experience with neuromuscular diseases. In some cases that led to preventable complications which could have been avoided. In others, the absence of reliable assessment, parents rushed their children to emergency rooms even if this was often not necessary. And they were worried it may happen again.

We therefore decided to move beyond the video-monitoring solution to explore the feasibility of an innovative home-basedadvanced-telemedicine platform to perform challenging remote physical evaluation in type 1 and 2 SMA patients. Although there are many commercially available tools that could have been theoretically used to perform remote physical evaluation, there were however some concerns related to their applicability to type1 and 2 SMA patients. The concerns were related to the chest and trunk appearance of the SMA patients as most children present with kyphoscoliosis and thoracic asymmetry, ribs crowding, thoracic organ displacement (Fig. 1), that are often associated with pulmonary functional impairment and impaired airway clearance $[8,9,10]$. This has become particularly true in type 1 infants who now generally survive beyond the first year but despite the functional improvements, still have variable degree of trunk hypotonia and weakness causing kyphoscoliosis that has become one of the key features of the emerging post treatment new phenotypes.

Because of these concerns, we aimed to explore the feasibility of one of the advanced telemedicine platforms and explore its possible application into a clinical SMA setting.

More specifically, we wished to explore feasibility, accuracy, and experience with home-based virtual physical evaluation, especially focused on challenging lung auscultation, versus traditional bed-side exam in type 1 and 2 SMA children with different degrees of scoliosis and chest asymmetry. Our expectation is to find an innovative solution to guarantee safety and access to care, reduce infection exposure, logistic and economic burden for families and improve disease management, during Covid-19 and beyond.

\section{Material and Method}

\subsection{Study Population}


We enrolled 23 consecutive children, aged 0-18 years, with type1 or 2 SMA, regularly followed at our Unit. The study is part of a larger natural history study on SMA approved by the Ethics Committee of our Institution, and a fully informed consent from the parents of each participant was obtained before the study.

For all the participating families, information on type of SMA, degree of neuroscoliosis/chest deformities, type of treatment, number of previous lung infections/ICU admissions, type of ventilation (non-invasive vs mechanical vs spontaneous breathing) were collected in a dedicated form.

\subsection{Digital Health Solution}

All families who agreed to participate in the study were offered the possibility to use a new device providing home-based virtual physical evaluations. Tytocare $\mathbb{R}$ is an all-in-one innovative digital health solution, FDA/CE approved, Class IIa medical device, designed to perform on-demand remote physical evaluations. The device mounts 3 different sensors that allow users to capture and share with their remote physician: ears-nose-throat (ENT) videos, skin images; heart, lung, bowel auscultations through a digital stethoscope; heart rate and body temperature via infrared touchless sensor. The device displays useful interactive pictures to guide parents through the lung evaluation but only shows traditional auscultation landmarks, four on the back and four on the front.

\subsection{Lung Ultrasound point of care}

Lung Ultrasound (LUS) was performed to assess optimal lung auscultation landmarks in children with different degrees of scoliosis and chest asymmetry and to detect areas of atelectasis, air trapping, pleural effusion, lung consolidations and other anomalies.

The ultrasound machine ESAOTE MyLab 40, complies with the Medical Device Directive (MDD) $93 \backslash 42$ $\backslash$ EEC and subsequent amendments. In accordance with this directive, Esaote has classified it as Class IIa devices. The preset used was small parts. Linear probe $(12-6 \mathrm{MHz})$ was used in preschool children. In older children, we used a curved probe $(8-5 \mathrm{MHz})$. The focus was always positioned at the level of the pleural line. Images and clips were stored and archived. The ultrasound scans were performed by pediatricians and pediatric residents with certifications and / or with an experience of at least 3 years of practice with LUS.

The scans were made by investigating the anterior, lateral and posterior regions of the bilaterally thorax and placing the probe transversely and longitudinally along the lines considered traditional ultrasound findings: the parasternal line, the axillary line and the paravertebral line so as to fully explore the chest wall according to a methodical scheme previously described [11]. To investigate the anterior and lateral lung fields, patients were positioned, according to age, in a seated or supine position. The posterior lung fields have been explored in lateral decubitus and in sitting position.

\subsection{Study method}

We designed a two-steps, pilot study to compare home-based virtual care vs. in-person physical evaluation. Two different units: a "bed-side" and a "remote" evaluation team were recruited.

The study was divided in three phases:

\section{Identification of the auscultatory landmarks in SMA patients and concordance with the standard landmarks}

Children were first assessed during one of the routine consultations by a pediatric neurologist with expertise in SMA, one pediatrician expert in digital health and one pediatric ultrasonographer who were in charge of locating optimal lung auscultation landmarks for each child with different methodologies. Each of them examined the children separately and located what she/he believed to be optimal chest auscultation landmarks after evaluating with either traditional stethoscope or lung ultrasound point of care (Fig. 2). According to her/his expertise, each of the examiners filled the medical reports for each patient, drawing an individual map of auscultation landmarks and specifying any additional respiratory (vesicular murmur, crackle, rhonchus, wheeze, pleural rub or stridor) or sonographic findings. 
The landmarks identified by the multidisciplinary team were compared to the standard auscultation landmarks, four on the back and four on the front, provided by the device for patients with typical configuration of chest and trunk in the absence of spinal deformities.

Fig. 2 Identification of optimal chest auscultation landmarks

\section{Training sessions and application of the new device by carers}

The medical part of the "remote" team gave a 30 minutes in-person practical training. The carers were shown how to place the device following the map of optimal landmarks identified for each child and to perform lung auscultation while an ICT expert offered technical support for the whole team. After training, as soon as they felt confident, they autonomously placed the Tytocare digital stethoscope at the corresponding landmarks, perform the entire respiratory evaluation back and front on their own, made sure each lung recording was as clear as possible without background noise (the device provide such features) and forward the files. In case of poor quality recording sessions the device displays on its screen several alert messages inviting the examiner to repeat the step until acceptable quality is obtained before moving forward. At the end of the session parents alone or with their children, recorded one or more free short videos to describe their experiences, ease-of -use, difficulties, expectations and so on.

\section{Assessment of accuracy and sensitivity of the device in our cohort of SMA children.}

Data obtained by the carers were reviewed by an independent pediatric team who blindly accessed the encrypted patient's charts. The blinded team examined the lung sounds from each recorded station (landmarks) using high quality headset in a quiet room and filled a report assigning an overall quality value, from 1 (very poor) to 5 (very good), identify and report possible interfering/background noise (mechanical ventilation, heart sounds, voices, rustle, $\mathrm{TV} /$ music) for each of the auscultation landmark

The blinded team also compared the recordings of respiratory findings obtained using the device by the carers with the reports of the bedside team who used conventional auscultation techniques.

\subsection{Statistical analysis}

Inter-correlation coefficient was used to establish inter-observer reliability as part of the development of the study.

\section{Results}

We enrolled 23 SMA children. Their age range between 6 months and 18 years. Eight of the $23(35 \%)$ were affected by type 2 SMA and the other $15(65 \%)$ by type 1 . In agreement with the Dubowitz decimal classification [12], 2 (13\%) of the 15 type 1 SMA patients were classified as 1.1, $8(53 \%)$ as 1.5 and $5(33 \%)$ as 1.9 .

Three of the $23(13 \%)$ had no scoliosis (Cobb angle $<20 \mathrm{deg}), 12 / 23(52 \%)$ were classified as mild or moderate (Cobb angle between 20deg and 50deg), while 8/23 (35\%) showed severe scoliosis ( Cobb angle > 50deg) complicated by different degree of kyphosis or rotoscoliosis (Tab. 1).

Five of the $23(22 \%)$ were on spontaneous breathing, $12 / 23(52 \%)$ used non-invasive ventilation less than 16 hours/daily, none of them used non-invasive ventilation more than 16 hours/daily and 6/23 (26\%) were mechanically ventilated through tracheostomy (Tab. 1).

\section{Identification of the auscultatory landmarks in SMA patients and concordance with the standard landmarks}

The identification of landmarks using a traditional stethoscope showed high concordance between examiners $(100 \%), I C C=1$. The landmarks identified through clinical auscultation using a traditional stethoscope and lung ultrasound assessment also had high concordance (100\%) in all patients, irrespective of their scoliosis or chest deformities. Lung vertical artifacts, sub-pleuric consolidation and small atelectasis were quite common US findings, as confirmed by literature but did not interfere with lung auscultation. This allowed to draw a lung auscultation map for each patient with 8 different sites, 4 on the back and 4 on the front, with 
children-friendly an-allergic colored stickers, placed directly on the children's body (Fig. 2, Fig. 3). Parents were encouraged to take as many pictures as they wanted for future independent evaluations.

The landmarks identified through auscultation and LUS were identical to the standard landmarks provided by the device in 7 of the $23(30 \%)$, in another $8(35 \%)$ were located in proximity to the standard ones (within 2 to $5 \mathrm{~cm}$ circular area) while in the remaining $8(35 \%)$ resulted in completely different areas.

The concordance between personalized auscultatory and standard landmarks was higher in the non-sitters, i-e the children with the most severe phenotypes who can be only assessed in the supine position and can be postured to at least partially correct scoliosis, when present (table 1). The concordance was much lower in children with Cobb>50deg and chest deformities irrespective of the SMA type.

\section{Training sessions and application of the new device by carers.}

Once the auscultation landmarks were identified, the use of the device by the carers was very easy and fast. All carers reported that they found very useful that device showed notification of under-optimal recording and gave advice to improve exam quality reducing environment noise like talking or TV sounds or reduce pressure that may create annoying friction sounds between the digital stethoscope and the skin, likewise on a traditional stethoscope.

\section{Assessment of accuracy and sensitivity of the device in our cohort of SMA children.}

The audio files recorded by carers with the Tytocare digital stethoscope resulted clear when listened to remotely. The overall quality value assigned by the remote team was "very good" ( $5 / 5$ points). The vesicular murmur was detectable and rated as "very clear" in $20(87 \%)$ children out of $23(\%)$ and 3/23 (13\%) was "slightly disturbed" due to background noise (same as digital stethoscope friction, TV, voices). The remote team did not rate as "highly disturbed" none of the recordings.

All our patients had normal respiratory findings and these were concordant between the "bed-side" multidisciplinary teamconventional auscultation and the remote assessment in all cases.

\section{Discussion}

For children affected by type 1 and 2 SMA, advanced telemedicine platforms seem to be a feasible and accurate solution that represents an unanticipated positive experience to overcome old and new limitations in time of Covid.

Our study showed that lung auscultation in type 1 and 2 SMA children can be remotely performed even by layman parents. The feasibility of the tool in patients with SMA revealed that the tool can always be used to obtain reliable auscultation but adaptations to find optimal landmarks are often needed in cases with asymmetrical or rotated chest and trunk.

In our experience, remote auscultation resulted almost straightforward in children with symmetric chest or mild-moderate scoliosis. In contrast, in patients with severe scoliosis optimal landmarks for digital lung auscultation could still be identified but required a preliminary assessment by experienced physicians, with traditional stethoscopes that allowed a tailor-made mapping of the landmarks for future reference to be used at home by the carers. In this study we also used lung ultrasound but it did not provide additional information on the landmarks compared to the traditional stethoscope auscultation. Once the landmarks were identified, after adequate 30 minutes in-person training, all carers were able to perform the lung examination following the step-by-step procedure as displayed by the device. The carers of children with severe scoliosis (Cobb angle $>50 \mathrm{deg}$ ), roto-scoliosis, kyphosis or chest deformities, reported that extra care was required to firmly hold in place the digital device.

Our results would therefore suggest, that following some preliminary work for landmarks identification, the device can be easily used by carers. As several factors, such as growth, increasing scoliosis or chest deformities due to the progression of the disease may interfere with the position of the landmarks, we expect that the landmarks should be reassessed at each hospital visit 
In our study, recording good quality sounds did not represent a challenge for most carers as confirmed by the remote assessment of their recordings by the expert medical team. Obtaining good results was probably facilitated by the device displaying several alert messages when poor quality sounds were recorded, inviting the examiner to record again until acceptable quality was obtained. There were very few disturbed recordings that had minor issues rated by examiner as due to interfering noises such as friction (unsteady holding in place the device) or human voices heard in the background.

Interestingly, in the 5 patients who were ventilated, the sound of mechanical ventilation did not interfere with auscultation and was correctly recognized in $100 \%$ of cases, probably due to its characteristic noise and unchanged rhythm. Surprisingly, it did not even trigger an alert message from the device which would have prevented remote recording in these children.

When asked to comment on their experience in video, all carers reported an overall enthusiastic experience, with "surprising" "unexpected" and "easy" being the 3 more frequently used adjectives. When asked about the potential future implementation "hope" was the most common comment followed by "I wish I'll not make mistakes" apparently revealing the mixed feelings between excitement and anxiety. Some carers revealed some auscultation-related-stress which waned over time, with exercise, and with remote assistance by our trained physicians and by the ICT group. In all cases a carer's personal motivation contributed to the fast learning and to the high number of successful lung evaluations, regardless of previous technical skills, age, gender or scientific background.

As expected, having their carers performing the assessment was very well accepted and perceived as a lowstress procedure for children. The application of colorful stickers was perceived as a fun game, according to their carers opinion. We believe that such "hands-on" training could be scheduled as part of the routine clinical evaluation in neuromuscular centers.

One of the limitations of this study, that was meant to assess the suitability of the new devices, was that while we were able to establish the feasibility of the tool to obtain reliable recordings and to establish the adaptations needed and the level of training, we could not assess the sensitivity of the device to detect pathological findings, probably due to Covid-related restrictions and parental extra precautions, all the patients enrolled in this study were all in good health and were evaluated in the absence of lung infections. Further studies are therefore needed to evaluate the sensitivity of the device and the concordance of responses in comparison with traditional auscultation in neuromuscular patients affected by respiratory infections. In conclusion the results of our study, that is part of a larger Advance Telemedicine project, suggest, for the first time, that remote devices may be used to address the un-meet need reported by patients with chronic disabilities (such SMA) and their carers regarding the possibility to assess patients in the presence of a possible acute event. Although the use of the new device has been driven by the needs at the time of pandemic, their use could be postulated also in the future as they may facilitate some aspects of care, reduce the number of visits to the hospital and ease direct costs (i.e. transport, overnight stays) and indirect costs (work and school permits or organizational expenses) for families.

$(3103 \mathrm{w})$

\section{Author's contributions:}

DK, BB, MP, EM: Conceptualization, Formal Analysis, Investigation, Methodology, Writing Original Draft Preparation. DL, CP, RO, MCP: Data Curation, Formal Analysis, Investigation. DB, CdR: Data Curation, Formal Analysis, Methodology. AC, EM, PS, GS: Methodology.

\section{References}

[1] D’Amico A, Mercuri E, Tiziano FD, Bertini E. Spinal muscular atrophy. Orphanet J Rare Dis. 2011;6:71.

[2] Mercuri E, Finkel RS, Muntoni F, Wirth B, Montes J, Main M, et al. Diagnosis and management of spinal muscular atrophy: Part 1: Recommendations for diagnosis, rehabilitation, orthopedic and nutritional care. Neuromuscul Disord. 2018;28(2):103-15. 
[3] Finkel RS, Mercuri E, Meyer OH, Simonds AK, Schroth MK, Graham RJ, et al. Diagnosis and management of spinal muscular atrophy: Part 2: Pulmonary and acute care; medications, supplements and immunizations; other organ systems; and ethics. Neuromuscul Disord. 2018;28(3):197-207.

[4] Finkel RS, Mercuri E, Darras BT, Connolly AM, Kuntz NL, Kirschner J, et al. Nusinersen versus Sham Control in Infantile-Onset Spinal Muscular Atrophy. N Engl J Med. 2017;377(18):1723-32.

[5] Mendell JR, Al-Zaidy S, Shell R, Arnold WD, Rodino-Klapac LR, Prior TW, et al. Single-Dose GeneReplacement Therapy for Spinal Muscular Atrophy. N Engl J Med. 2017;377(18):1713-22.

[6] Pane M, Coratti G, Sansone VA, Messina S, Bruno C, Catteruccia M, et al. Nusinersen in type 1 spinal muscular atrophy: Twelve-month real-world data. Ann Neurol. 2019;86(3):443-51.

[7] Mercuri E, Zampino G, Morsella A, Pane M et al., Contactless: a new personalised telehealth model in chronic pediatric diseases and disability during the COVID-19 era. Italian Journal of Pediatrics (2021) 47:29 https://doi.org/10.1186/s13052-021-00975-z

[8] Sansone VA, Pirola A, Albamonte E, Pane M et al. Respiratory Needs in Patients with Type 1 Spinal Muscular Atrophy Treated with Nusinersen. J Pediatr. 2020 Aug;223:227-228. doi: 10.1016/j.jpeds.2020.04.069. Epub 2020 May 4.

[9] Agnieszka S, Mazurkiewicz L, Maślanko K, Rekowski W and Jędrzejowska M. Cervical rotation, chest deformity and pelvic obliquity in patients with spinal muscular atrophy. BMC Musculoskelet Disord. 2020; 21: 726. Published online 2020 Nov 7. doi: 10.1186/s12891-020-03710-7. PMCID: PMC7648994. PMID: 33160358

[10] Trucco F, Scoto MC, Coratti G, Main ML et al. Respiratory Trajectories in Type 2 and 3 Spinal Muscular Atrophy in the iSMAC Cohort Study. Neurology 2021; 96:e587-e599. doi: 10.1212/WNL.0000000000011051

[11] Copetti R, Cattarossi L. Ultrasound diagnosis of pneumonia in children. Radiol Med. 2008; 113(2):190198.

[12] Dubowitz V. Chaos in classification of the spinal muscular atrophies of childhood. Neuromuscul Disord $1991 ; 1: 77-80$.

Fig. 1 Examples of chest deformities in SMA patients

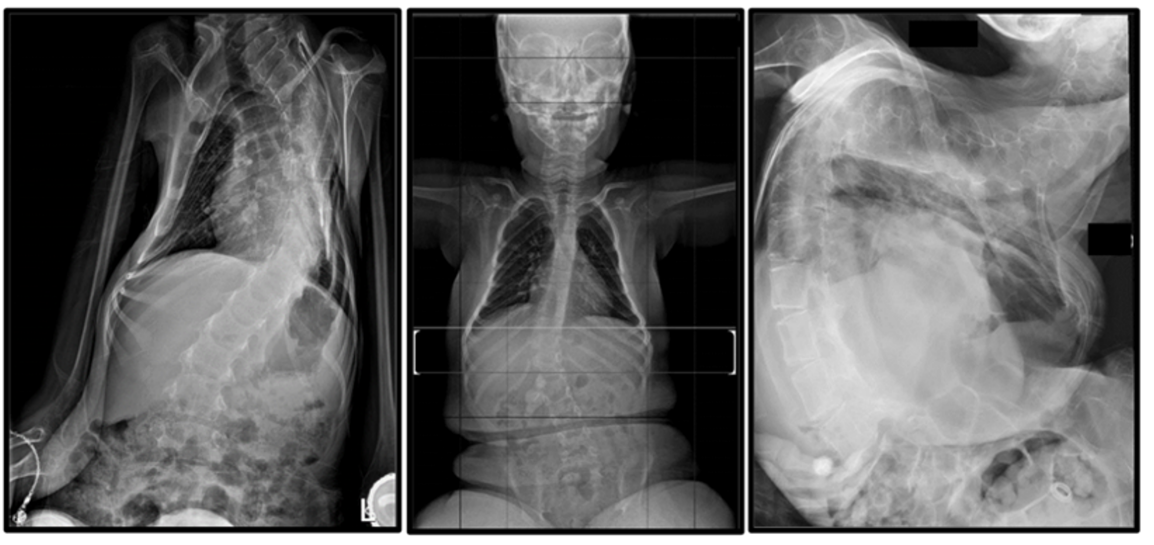


Fig. 2 Identification of optimal chest auscultation landmarks
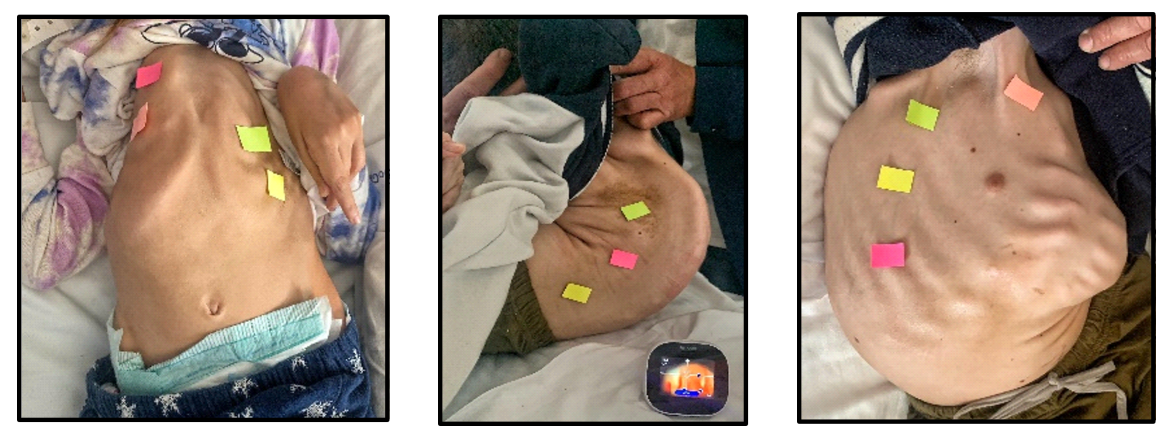

Fig 3 Cares recording breathing sounds on identified auscultatory landmarks
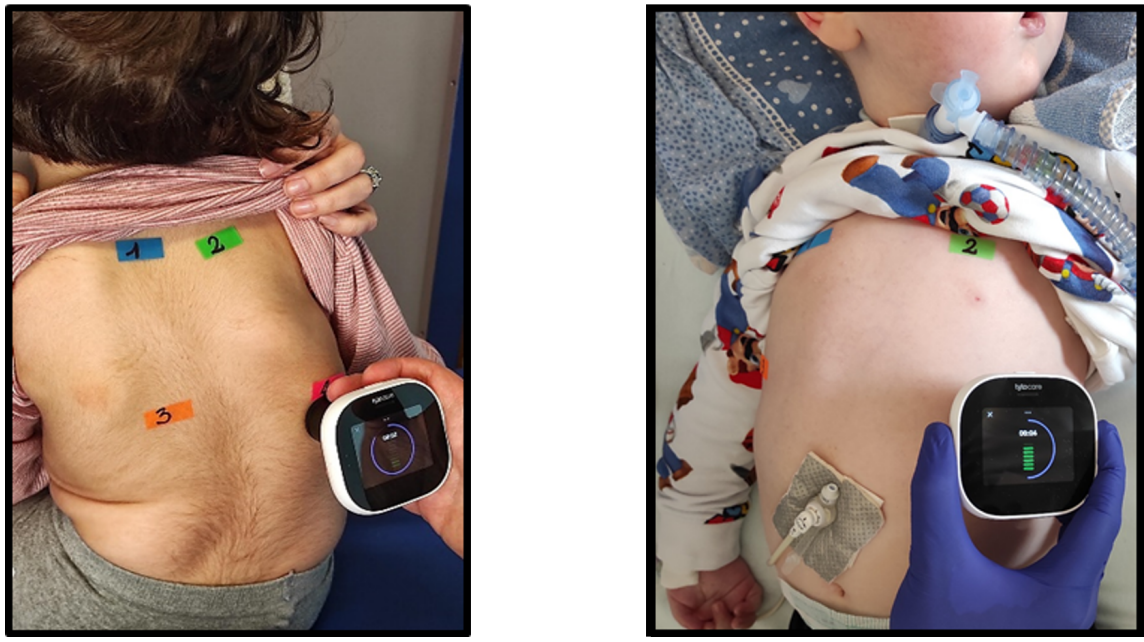

\section{Hosted file}

SMA TYTO tabella 1.docx available at https://authorea.com/users/437149/articles/538989-frombed-side-to-web-side-advanced-telemedicine-platform-for-spinal-muscular-atrophy 